\title{
Interactive comment on "A multi-model intercomparison of halogenated very short-lived substances (TransCom-VSLS): linking oceanic emissions and tropospheric transport for a reconciled estimate of the stratospheric source gas injection of bromine" by R. Hossaini et al.
}

Anonymous Referee \#2

Received and published: 14 February 2016

General

This paper presents a comprehensive model intercomparison of the impact of bromine containing VSLS on the stratospheric bromine loading. This is a good initiative and the outcome of this intercomparison will be important of assessing the impact of bromine on stratospheric ozone. It is in particular noteworthy that a lot of observations are employed to assess the quality of the model results.

Printer-friendly version

Discussion paper 
I have some points (see below), where I think the discussion in the paper can be clarified and improved. The impact of particular model features (e.g., the convective schemes employed) on the different results could be brought out more clearly. The reader ultimately will be interested in what the problematic model features are, because these are the features that need improvement in the further developments of such models. This point cold be brought across in the paper in a better way.

Interactive

In summary, I think that a revised version of the paper, taking into account the points raised in the reviews will be a valuable contribution to ACP.

\section{Detailed comments}

Five out of the 11 participating models are nudged to or driven by ERA-Interim. While ERA-Interim is a good choice, this fact will lead to the multi-model mean being biased to an ERA-Interim world. I suggest to bring this point across more clearly. Does this fact have any implications for the conclusions of this model intercomparison?

Another model feature, which is important for tropospheric transport of VSLS is the convective parametrisation used in the model (see for example Rybka, $\mathrm{H}$. and Tost, $\mathrm{H}$.: Uncertainties in future climate predictions due to convection parameterisations, Atmos. Chem. Phys., 14, 5561-5576, doi:10.5194/acp-14-5561-2014, 2014, and references therein). I suggest more discussion of this point in the paper. Also, the information of the convective scheme used in the different models should be included in Table 2. Perhaps some of the model differences and some of the model similarities can be attributed to using a particular convective parametrisation or a particular meteorology? I also have reservations about the concept of a "preferred" tracer. I think this means that the emission inventory somehow interacts with the transport scheme of the model to produce reasonable results at higher altitudes. But this means that the higher altitude agreement could be right for the wrong reason. I know it is demanding a lot from 
models, but of course one would expect to design independently the best emission inventory and the best (vertical) transport to obtain the best agreement with measurements. Obviously this model intercomparison cannot achieve this goal, but I think the discussion of these issues could be improved.

Finally, the impact of ENSO activity on the stratospheric bromine loading is unclear. What is the message of the paper here? The paper states that there is a strong correlation of SGI with ENSO (e.g. abstract), but that there is no correlation of ENSO (MEI) with the bromine loading in the LS (e.g. conclusions). But SGI is important for the bromine loading in the LS. This points needs to be clarified and better discussed in the paper.

\section{Minor issues}

- Title: I am not sure if "TransCom-VSLS" should be in the title; the name of the project will not be relevant on a timescale of years, when the paper will still be read.

- I. 7: I do not think that model estimates should be used to "constrain" measurements.

- line 20: change 'optimal' to 'best'

- I. 36: Isn't 6 month a bit long for very short lived?

- 151: 'recent' twice in this sentence

- I. 52: try \mathrm\{VSLS $\}$ to avoid italics in VSLS. (Similar for MAPE (I. 345) below).

Printer-friendly version

Discussion paper

- I. 59: 'owing to' instead of 'due to' 
- I. 76: I think you mean Tissier and Legras here

- I. 78: do you mean "broadly similar" here?

- I. 100: what do you mean by "climate modes" - more explanation here.

- Figure 1: This figure is not really discussed in the paper. Which message does it communicate? I suggest removing the figure from the paper.

- I. 144: is a bottom-up ...

- line 179: this means that the multi-model mean is highly influenced by CTMs driven by ERA-Interim data - correct?

- I. 211: instead of 'see also' you could perhaps state for which information which paper should be consulted.

- I 301: what is the reason that 'clear outliers' are found? Are these models with obvious errors?

- I. 329: use $r$ for the correlation coefficient

- I. 366: why does convection influence "near-surface" abundances of VSLS?

- I. 414: I think it is problematic that models have a preferred tracer: doesn't this imply that results could be right for the wrong reason?

- I. 425: Where is the reproduction of the c-shape shown? This seems an important issue.

- I. 435: The concept of a 'preferred' tracer means that the emission inventory somehow interacts with the models transport scheme to produce reasonable results at higher altitudes - correct? Can you describe in more detail here, what 'worse agreement' means? 
- I. 485: is CO really short-lived?

- I. 492: state the lifetime in months/weeks

- I. 527: you might want to add here also Tissier and Legras 2015; Vogel et al. 2014

- I 560: Clarify which best estimate is meant here, TransCom or WMO.

- I. 593-595: The last sentence states that the VSLS loading in the LS is not correlated to $\mathrm{MEI}$. But the sentences above state that bromine SGI is sensitive to modes such as MEl. Isn't this a contradiction? I think more discussion is require here.

- I. 598: change to: these processes

- I. 599: change 'a range' to 'a number'

- I. 614-618: Is the point here that the seasonal cycle is not dependent on the emission inventory, but the absolute model-measurement agreement is? How can this be the case. Please clarify. (See also abstract).

- I. 626: change optimal to best

- I. 634: what exactly is meant by 'online calculations'?

- I. 648: But the 'higher altitudes' are most relevant for the transport of VSLS into the stratosphere - correct?

- I. 663: You mean the SGI range by Carpenter and Reiman, add the citation for clarification.

- I. 670-672: This is astonishing, isn't it? I suggest somewhat more discussion on this point. 
- I. 676: change 'changes to' to 'changes of'

- I. 678: change 'increased' to 'increase of the'

- I. 679: distinguished from what?

- I. 689: why is R Hommel not abbreviated?

- Fig. 1: not sure if this figure is necessary

- Fig. 2: Continents in light grey would look better than in black.

- References: There are some references that need to be updated; ACP vs ACPD, Werner et al., 2016 etc.

Interactive comment on Atmos. Chem. Phys. Discuss., doi:10.5194/acp-2015-822, 2016. 